\title{
The establishment of the objective diagnostic markers and personalized medical intervention in patients with major depressive disorder: rationale and protocol
}

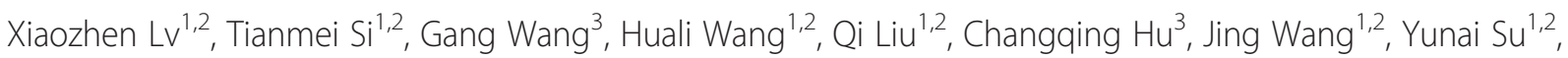
Yu Huang ${ }^{4}$, Hui Jiang ${ }^{4}$ and $X$ in $Y_{u^{1,2^{*}}}$ (i)

\begin{abstract}
Background: Major depressive disorders (MDD) is a common mental disorder with high prevalence, frequent relapse and associated with heavy disease burden. Heritability, environment and their interaction play important roles in the development of MDD. MDD patients usually display a wide variation in clinical symptoms and signs, while the diagnosis of MDD is relatively subjective. The treatment response varies substantially between different subtypes of MDD patients and only half respond adequately to the first antidepressant. This study aims to define subtypes of MDD, develop multi-dimension diagnostic test and combined predictors for improving the diagnostic accuracy and promoting personalized intervention in MDD patients.

Methods/Design: This is a multi-center, multi-stage and prospective study. The first stage of this study is a case-control study, aims to explore the risk factors for developing MDD and then define the subtypes of MDD using 1200 MDD patients and 1200 healthy controls with a set of questionnaire. The second stage is a diagnostic test, aims to indentify and replicate the potential indicators to assist MDD diagnosis using 600 MDD patients and 300 healthy controls from the first stage with a set of questionnaire, neuropsychological assessment and a series of biomarkers. The third stage is a 96-week longitudinal study, including 8-week acute period treatment and 88-week stable period treatment, aims to identify overall predictors of treatment effectiveness on MDD at week 8 post treatment and to explore the predictors on MDD prognosis in the following 2 years using 600 MDD patients from the first stage with a set of questionnaire, neuropsychological assessment and a series of biomarkers. The primary outcome measure is the change of the total score of 17-Item Hamilton Rating Scale for Depression.
\end{abstract}

Discussion: This study will provide strong and suitable evidence for enhancing the accuracy of MDD diagnosis and promoting personalized treatment for MDD patients in clinical practice.

Trial registration: ClinicalTrials.gov: NCT02023567; registration date: December 2013.

Keywords: Major depressive disorder, Subtype, Diagnostic, Personalized treatment

\footnotetext{
* Correspondence: yuxin@bjmu.edu.cn

${ }^{1}$ Peking University Sixth Hospital (Institute of Mental Health), Beijing, China

${ }^{2}$ National Clinical Research Center for Mental Disorders \& Key Laboratory for

Mental Health, Ministry of Health, Peking University, Beijing, China

Full list of author information is available at the end of the article
} 


\section{Background}

Major depressive disorders (MDD) is a common, complex, often difficult-to-treat and high-relapse clinical condition [1]. MDD causes the largest amount of disability, accounting for almost $12 \%$ of all total years lived with disability worldwide [2]. MDD was also a contributor of burden allocated to suicide and ischemic heart disease [3]. The pain and suffering of individuals with MDD and those close to them result in a heavy economic toll to this country in terms of both treatment costs and lost productivity [4]. China is also confronted with this daunting challenges against MDD. It was assessed that the 1-month prevalence of mood disorders (mainly MDD) was $6.1 \%$ [5].

MDD commonly arises when a vulnerable individual confronts adversity. MDD is familial, with heritability estimated to be 0.37 . Environmental influences specific to an individual are also etiologically significant $[6,7]$. Genetic factors partially influence overall risk of illness, but also influence the sensitivity of individuals to the depression-inducing effects of environmental adversity. The interaction of genotype and environment is significant in the prediction of onset of MDD [8]. Despite decades of research, there remains little consensus on how to distinguish between MDD subtypes. Loo et al. examined the evidence for the existence of data-driven symptomatic subtypes of depression and did not provide conclusive evidence for the existence of depressive symptom dimensions or symptomatic subtypes [9]. A persistent theme in the debate on the classification of MDD has been the question of how to distinguish biological depressions from depressions that are social in origin. It appears to be " at least partly" to distinguish those individuals whose depressive illness is largely "genetic" versus "environmental" [10]. One the other side, some distinct subtypes had been suggested and found subtypes associated with treatment outcomes [11-13]. For optimized treatment, it is probably meaningful to classify MDD into different subtypes basing on its psychopathology.

The diagnosis of MDD is based on relatively subjective interviews and questionnaires for assessments of symptoms. However, affected individuals display quite a wide variation in clinical symptoms and signs [10]. Even in some high-income countries, people who are depressed are not always correctly diagnosed [1]. Biomarkers in psychiatry present a promising addition to advance the diagnosis, treatment and prevention of psychiatric diseases [14]. The development of reliable diagnostic tests using biomarkers could aid in the diagnosis of MDD. Many peripheral biomarkers, including inflammatory cytokines, immunological markers, growth factors, endocrine factors, metabolic markers and oxidative stress markers, have been investigated because these markers are thought to be involved in the pathophysiology of MDD [14-18]. However, it is clear that even though a large number of biomarkers have been linked to MDD, each individually explains a very modest proportion of the variance in MDD risk. For clinical diagnosis of MDD, all individual marker-based approaches yielded insufficient sensitivity and specificity [19]. It had been reported that a composite, multi-assay diagnostic test for MDD demonstrated adequate sensitivity and specificity (about 91 and $81 \%$ ) for American [19]. The results in a Japanese population were not in conformity with those in American [20]. It is needed to confirm the performance of the test in large Chinese sample.

Several treatments for MDD are available, while response to treatments varies substantially between patients and more than half will fail to respond adequately to the first antidepressant they are prescribed [21]. The heterogeneity of treatment effects complicates clinical decision-making. One approach to enhancing treatment outcomes in MDD has been the use of standardized sequential treatment algorithms and measurement-based care. Pretreatment tests that predict which patients will respond to which types of treatment could save time, money and patient burden. A large body of literature reported that some demographic characteristics, clinical symptoms, stress events, genotypes and biomarkers could foretell overall treatment outcome [11-13, 15, 22-37]. However, robust and combined predictors of treatment response remain elusive $[7,38]$. It is urgent and critical to combine possible factors to form the basis of new paradigms for antidepressant treatment selection.

\section{Objectives}

This study is designed to verify the role of heritability and environment in the onset of MDD, define the subtypes of MDD and evaluate a range of factors/predictors to aid MDD diagnosis and personalized treatment within MDD patients and healthy controls. The aims of this study are to:

1. Verify the role of heritability and environment in the onset of MDD and define the subtypes of MDD according to the influence of heritability and environment on its development.

2. Identify potential indicators to assist MDD diagnosis basing on the baseline information, neuropsychological assessment and biological markers.

3. Identify overall predictors for treatment effectiveness of selective serotonin reuptake inhibitors (SSRIs) on MDD after up to 8 weeks

4. Explore the predictors on MDD prognosis in the following two years. 


\section{Methods/Design}

\section{Organizational structure and quality control}

The infrastructure of this study includes an executive management team, 9 clinical sites at 9 top tertiary hospitals (7 within academic settings and 2 in clinical practices) located in 6 provinces/municipalities and one clinical research organization. Each clinical site has a principal investigator (responsible for all work in this site), one research coordinator (responsible for the coordination in this site/with the executive management team), 2 to 4 investigators (responsible for recruitment, clinical evaluation, neuropsychological assessment, blood specimen collection), one data typist (responsible for entering paper-based questionnaire data into a web-based data system) and one clinical research assistant (CRA) assigned by the clinical research organization (responsible for supervising compliance to study protocol and checking the conformance of the paper-based questionnaire and the web-based).

Each clinical sites are selected based on the likelihood of executing the protocol and their previous research experience. All principal investigators have chaired or participated in some clinical studies. Prior to enrolling participants in each site, work manuals will be supplied to each site to provide instructions on all relevant research issues, including enrollment, clinical evaluation, neuropsychological assessment, serious adverse events reporting procedure, data entering and checking and blood specimen collection, storage and transportation. The researchers are all strictly trained according to the work manual. As a new staff joins in, they will be trained by their principal investigator and research coordinator. Clinical data are acquired by psychiatrists. The investigators will remain in contact with those participants who should be followed up to minimize premature discontinuation. The CRA will visit the research site once a week and feedback the progress and main problems to the principal investigator and the executive management team. A monthly review of the program progress, key problems and advice will be reported to all researchers by the executive management team. In addition, a group of experts assigned by the executive management team will go to each site twice a year to guide and confirm the protocol implementation and assess the reliability of clinical evaluation. Inter-rater reliability for the primary outcome measure (the 17-Item Hamilton Rating Scale for Depression, $\mathrm{HRSD}_{17}$ ) [39] is audited for each psychiatrist who involved in clinical evaluation at each site annually. The executive management team calls for all principal investigators and research coordinators once a year face-to-face to discuss the difficulties and share experience to ensure the protocol implementation and study quality.

\section{Study design}

This is a multi-center, multi-stage and prospective study. It includes 3 stages. (1) The first stage is a case-control study, basing on the baseline information of $1200 \mathrm{MDD}$ patients (1200-MDD patients group) and 1200 health controls (1200-Healthy controls group), aims to explore the risk factors of developing MDD and then define the subtypes of MDD. (2) The second stage is a diagnostic test, basing on the baseline information, neuropsychological assessment and biological markers of $600 \mathrm{MDD}$ patients (600-MDD patients subgroup) and 300 health controls (300-Healthy controls subgroup) from the first stage, aims to indentify and replicate the potential indicators to assist MDD diagnosis. (3) The third stage is a 96week longitudinal study, including 8-week acute period treatment and 88-week stable period treatment of the 600-MDD patients subgroup. A total of 600 MDD patients from the first stage, who would receive one of 6 SSRIs within the range of does for 8 weeks (fluoxertine hydrochloride 20-60 mg/day, paroxetine hydrochloride 20-60 mg/day, sertraline hydrochloride 50-200 mg/day, citalopram 20-60 mg/day, escitalopram 10-20 mg/day, fluvoxamine $50-300 \mathrm{mg} /$ day) decided by their attending physicians, would enter into the second and third stage. The 600-MDD patients subgroup would receive clinical evaluation at baseline, week 2,4 and 8 and receive neuropsychological assessment and blood collection at baseline and week 8 meanwhile. The aim of this phase is to identify overall predictors of treatment effectiveness of SSRIs, basing on the baseline information, clinical evaluation, neuropsychological assessment and biological markers of 600-MDD patients subgroup. The $600-$ MDD patients subgroup would enter into the stable period treatment and be followed up in the following 88 weeks after completing the above acute period treatment. The treatment in the stable period would be completely decided by their attending physicians according to clinical practice. These patients would receive clinical evaluation at week 24, 48 and 96 and receive neuropsychological assessment and blood collection at week 48 meanwhile. The aim of this phase is to explore the predictors for MDD prognosis.

\section{Participants}

The participants are planned to enroll between December 2013 to December 2016. The goal is to recruit 1200 MDD patients and 1200 healthy controls to complete baseline evaluation and 300 out of 1200 healthy controls will meanwhile receive neuropsychological assessment and blood collection. The 600-MDD patients subgroup (600 out of 1200 MDD patients), who meet more strict criteria and will receive one of 6 SSRIs for 8 weeks in acute period as aforementioned, will be followed up for 96 weeks. The details of inclusion and exclusion criteria for each group are listed in Table 1. 
Table 1 The inclusion and exclusion criteria for MDD patients and healthy controls, respectively

\begin{tabular}{|c|c|c|}
\hline Study group & Inclusion criteria & Exclusion criteria \\
\hline $\begin{array}{l}\text { 1200-MDD } \\
\text { patients }\end{array}$ & $\begin{array}{l}\text { (1) age between } 18 \text { and } 55 \text { years at the time of enrollment; } \\
\text { (2) diagnosis of MDD based on the Chinese Version of MINI } \\
\text { according to DSM-IV TR; } \\
\text { (3) first-episode or relapsed; } \\
\text { (4) having the ability of reading and writing to complete the } \\
\text { questionnaire and psychological assessment; } \\
\text { (5) providing written confirmation of informed consent. }\end{array}$ & $\begin{array}{l}\text { (1) lifetime or current diagnosis of other psychotic disorder, alcohol/ } \\
\text { substances dependence or cognitive impairment; } \\
\text { (2) severe somatic diseases, such as severe cardio-cerebral vascular } \\
\text { diseases, respiratory diseases, liver diseases, kidney diseases, or } \\
\text { malignant tumors; } \\
\text { (3) not signed the informed consent; } \\
\text { (4) been engaging in other studies. }\end{array}$ \\
\hline $\begin{array}{l}600-\mathrm{MDD} \\
\text { patients } \\
\text { subgroup }\end{array}$ & $\begin{array}{l}\text { Besides those same as } 1200-M D D \text { patients', more inclusion } \\
\text { criteria are as the follows: } \\
\text { (1) first-episode or relapsed more than } 1 \text { time in the past } \\
3 \text { years and in acute episode period; } \\
\text { (2) total score of } \mathrm{HRSD}_{17} \geq 14 \text { when being screened; } \\
\text { (3) not taking anti-depressant regularly in the past } 2 \text { weeks or } \\
\text { should change anti-depressant according to the } \\
\text { psychiatrist's advice. }\end{array}$ & $\begin{array}{l}\text { Besides those same as 1200-MDD patients', more exclusion criteria are } \\
\text { as the follows: } \\
\text { (1) resistant depression (not improved after taking } 2 \text { kinds of anti- } \\
\text { depressant with adequate dosage and duration; } \\
\text { (2) having history of epilepsy; } \\
\text { (3) taking MECT therapy in the past } 3 \text { months; } \\
\text { (4) pregnant or breast-feeding. }\end{array}$ \\
\hline $\begin{array}{l}\text { 1200-Healthy } \\
\text { controls }\end{array}$ & $\begin{array}{l}\text { (1) age between } 18 \text { and } 55 \text { years at the time of enrollment; } \\
\text { (2) providing written confirmation of informed consent prior } \\
\text { to engaging the study. }\end{array}$ & $\begin{array}{l}\text { (1) lifetime or current diagnosis of any mental diseases; } \\
\text { (2) severe somatic diseases, such as severe cardio-cerebral vascular } \\
\text { diseases, respiratory diseases, liver diseases, kidney diseases, or } \\
\text { malignant tumors; } \\
\text { (3) not signed the informed consent; } \\
\text { (4) been engaging other studies. }\end{array}$ \\
\hline
\end{tabular}

Abbreviations: MDD major depressive disorder, MINI mini-international neuropsychiatric interview, DSM-IV TR diagnostic and statistical manual of mental disorders, fourth edition text revision, $H R S D_{17}$ the 17-item Hamilton rating scale for depression, $M E C T$ modified electric convulsive therapy

\section{Enrollment, clinical treatment and follow-up}

1. For 1200 MDD patients, the potential participants of the outpatients and inpatients in each clinical sites would be screened and enrolled by the investigators according to the inclusion and exclusion criteria of 1200MDD patients firstly. To ensure representative and reflect the routine clinical practice, the treatment of eligible MDD patients would be decided and adjusted by their attending physicians. The $600-\mathrm{MDD}$ patients subgroup would receive the same type and dose of one of 6 SSRIs as aforementioned in the 8-week acute treatment period. In this period, except additional treatments for comorbid physical diseases or short-lasting benzodiazepines for severe insomnia, other antipsychotic medications, other anti-depressant, mood stabilizer, systematic psychotherapy and long-lasting benzodiazepines were not allowed. The $600-\mathrm{MDD}$ patients subgroup will receive clinical visits at 2nd, 4th, 8th, 24th, 48th and 96th week in the following 96 weeks after baseline evaluation. The primary treatment outcome evaluation index is the change of the total score of $\mathrm{HRSD}_{17}$ : treatment effective defined as a $\geq 50 \%$ decrease from the baseline total score of $\mathrm{HRSD}_{17}$, clinical recovery defined as a $\geq 50 \%$ decrease from the baseline total score of $\mathrm{HRSD}_{17}$ and the total score of $\mathrm{HRSD}_{17}<7$, no response defined as a $<50 \%$ decrease from the baseline total score of $\mathrm{HRSD}_{17}$. The secondary index includes the change of the total score of Hamilton Anxiety Scale (HAMA), Global Impression of Severity (CGI-S), number of MDD patients with serious adverse events. Other $600 \mathrm{MDD}$ patients will complete this study after baseline evaluation.
2. For 1200 healthy controls, except for the researchers involved in this study and the family members of enrolled MDD patients, those working in the clinical sites, the friends of the patients, college students, or the residents near the clinical sites are all potential participants. Those who are interested in this study will contact the investigators and be enrolled according to the inclusion and exclusion criteria of healthy controls. All healthy controls will receive baseline evaluation and 300 out of them will voluntarily receive neuropsychological assessment and supply blood specimen.

See "Fig. 1 the research flowchart" for a detailed overview of the research procedure.

\section{Data collection}

Screening, demographic, clinical and cognitive function data

At screening, the trained investigators gather participant eligibility. The Chinese Version of MINI [40] was used to confirm DSM-IV criteria for MDD [41], and assess for psychiatric and substance abuse disorders and other potential exclusion criteria. After enrollment, data of demographic, medical history, family history of psychiatric disease, MDD course, treatment process, concurrent treatment, stress events, mood disorder episodes and adverse events at baseline and/or follow-up point would be collected using a set of self-designed questionnaire; other data, including life events in the past year, adverse childhood experience, social support, coping style, personality trait, anxiety symptom, depressive symptom severity and improvement would be assessed 


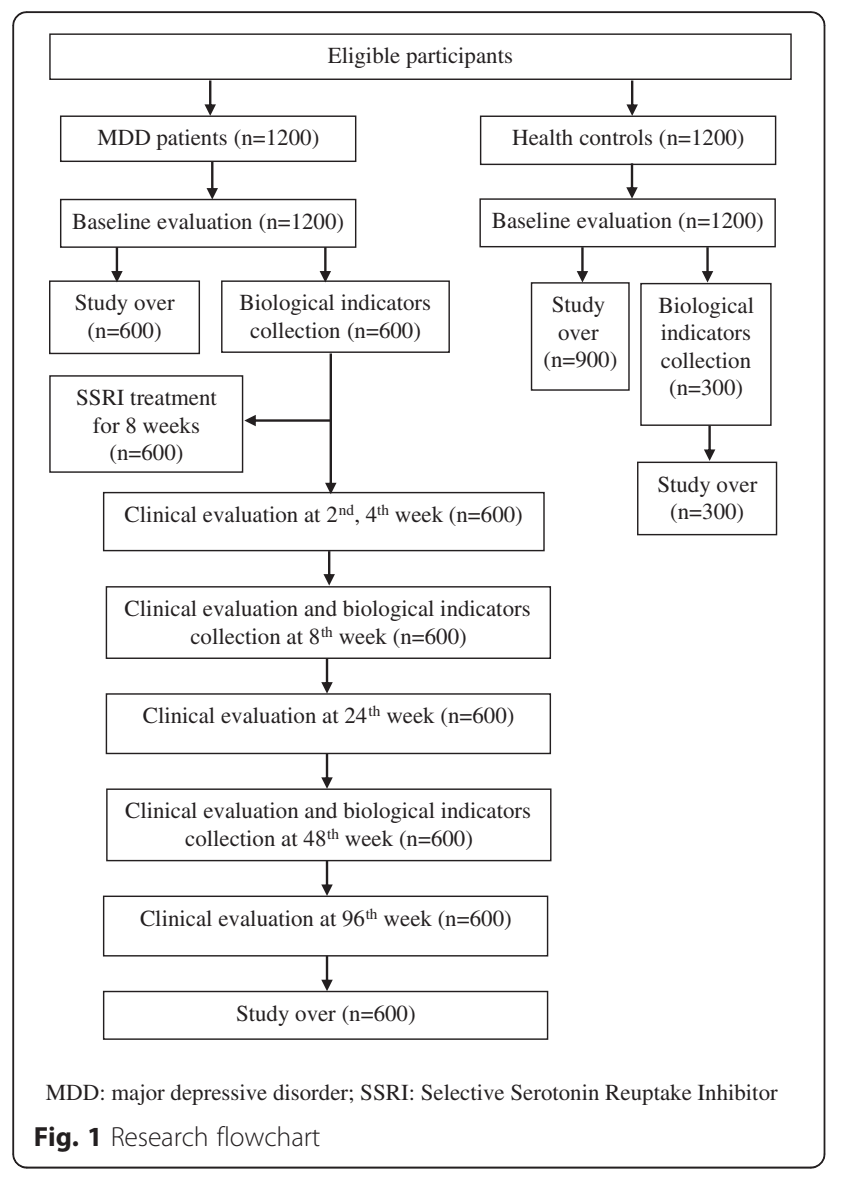

and acquired using standardized Chinese version of scales, respectively, including Life Events Scale, Childhood Trauma Questionnaire, Social Support Questionnaire, Simplified Coping Style Questionnaire and, Eysenck Personality Questionnaire, Hamilton Anxiety Scale, $\mathrm{HRSD}_{17}$ and Clinical Global impression Scale [39, 42].

A series of neuropsychological assessment [43] to reflect cognitive function will be completed at baseline for 600-MDD patients subgroup and 300-Healthy controls subgroup, respectively, and also at 8th and 48th week for 600-MDD patients subgroup, respectively. There are 5 domains and each is assessed by at least one test: attention/vigilance assessed by Continuous Performance Test-Identical Pairs, speed of information processing assessed by Animal Verbal Fluency Scale, Color Trial Test I and II and The Brief Assessment of Cognition in Schizophrenia, visual learning assessed by Brief Visual Memory Test-Revised, verbal learning assessed by Hopkins Verbal Learning Test-Revised and executive function assessed by Stroop Color Word Test. The trained investigator would guide the participants completing the tests according to standardized instructions. Except for the test of Continuous Performance Test-Identical Pairs completed using a standardized computerized touch screen platform, other tests would be completed using standardized scales guiding by the investigator.

The measure, participant, method, assessment time and administrator of the data collection are listed in Table 2.

\section{Molecular data}

Blood samples were collected by venepuncture at baseline for 600-MDD patients subgroup and 300-Healthy controls subgroup, respectively, and also at 8th and 48th week for 600-MDD patients subgroup, respectively. Plasma samples were collected in EDTA containing tubes; within $1 \mathrm{~h}$ from blood sampling, they will be centrifuged at $3000 \mathrm{rpm}$ for $15 \mathrm{~min}$. Afterward plasma samples were carefully transferred to new tubes and stored at $-80^{\circ} \mathrm{Cuntil}$ further analysis. Serum samples were collected in anticoagulant-free tubes. After centrifugation, serum will be drawn off and frozen at $-80^{\circ} \mathrm{Cuntil}$ needed. Avoid repeated freeze-thaw cycles. Assays will be performed at the central laboratory (Peking University Sixth Hospital). Immune/inflammation and neurotrophic factor pathways have been implicated in the pathogenesis of MDD. The activity of peripheral cytokines correlates with inflammatory processes in the central nervous system [32]. Immune and neurothrophic biomarkers that maybe associated with MDD will be measured in serum or plasma with commercially available kits following the manufacturer's instructions.

Genomic DNA will be extracted from venous blood using a commercially available QIAamp ${ }^{\circledR}$ DNA Blood Mini Kit. Based on the HapMap database [44] and the NCBI SNP database [45], we selected functional polymorphisms related to depression canidate genes with minor allele frequencies (MAFs) $>5 \%$ according to the HapMap database for CHB [44]. Functional polymorphisms will be determined using the F-SNP database [46] which identifies polymorphisms with effects on protein coding, splicing regulation, transcription regulation, and/or post translation. Genotyping will be conducted using the Sequenom MassArray system (Sequenom iPLEX assay) by following the manufacturer's instructions. Approximately $15 \mathrm{ng}$ of genomic DNA will be used to genotype each sample. Locus-specific PCR and detection primers will be designed using the MassArray Assay Design 3.0 software (Sequenom). The DNA samples will be amplified by multiplex PCR reactions, and the PCR products will then be used for locus-specific single-base extension reaction. The resulting products will be desalted and transferred to a 384-element SpectroCHIP array. Allele detection will be performed using MALDI-TOF MS spectroscopy. The mass spectrograms will be analyzed by the MassArray TYPER software (Sequenom). To assess genotyping quality, $3.5 \%$ of samples will be genotyped twice. 
Table 2 Data Collection at Screening, Baseline and Follow-up Evaluation

\begin{tabular}{|c|c|c|c|c|c|}
\hline Domain & Measure & Participant & Method & $\begin{array}{l}\text { Assessment } \\
\text { time }\end{array}$ & Administrator \\
\hline Informed consent & Informed consent & All participants & Interview & Screening & Investigator \\
\hline Eligibility & Inclusion/Exclusion & All participants & Interview & Screening & Investigator \\
\hline Psychiatric diagnoses & MINI & All participants & Interview & Screening & Investigator \\
\hline $\begin{array}{l}\text { Demographic, medical history, family history } \\
\text { of psychiatric disease and MDD course }\end{array}$ & $\begin{array}{l}\text { Self-designed } \\
\text { questionnaire }\end{array}$ & All participants ${ }^{a}$ & Self-report & Baseline & Investigator \\
\hline $\begin{array}{l}\text { Life events in the past year, adverse } \\
\text { childhood experience, social support, coping } \\
\text { style, personality trait }\end{array}$ & $\begin{array}{l}\text { LES, CTQ, SSQ, } \\
\text { SCSQ,EPQ }\end{array}$ & All participants & Interview & Baseline & Investigator \\
\hline $\begin{array}{l}\text { Treatment process, concurrent treatment, } \\
\text { stress events, mood disorder episodes and } \\
\text { adverse events }\end{array}$ & $\begin{array}{l}\text { Self-designed } \\
\text { questionnaire }\end{array}$ & $\begin{array}{l}\text { 600-MDD patients } \\
\text { subgroup }\end{array}$ & Self-report & Every follow-up & Investigator \\
\hline Anxiety symptom & HAMA & $\begin{array}{l}\text { 600-MDD patients } \\
\text { subgroup }\end{array}$ & Interview & Every follow-up & Investigator \\
\hline Depressive symptom severity & $\mathrm{HRSD}_{17}$ & $\begin{array}{l}\text { 600-MDD patients } \\
\text { subgroup }\end{array}$ & Interview & $\begin{array}{l}\text { Baseline and } \\
\text { every follow-up }\end{array}$ & Investigator \\
\hline Depressive symptom improvement & CGIS & $\begin{array}{l}\text { 600-MDD patients } \\
\text { subgroup }\end{array}$ & Interview & Every follow-up & Investigator \\
\hline Cognitive function & $\begin{array}{l}\text { CPT-IP, AVFSS, CTTI and } \\
\text { II, BACS, BVNT-R, } \\
\text { HVLT-R, SCWT }\end{array}$ & $\begin{array}{l}\text { 600-MDD patients and } \\
\text { 300-Health controls } \\
\text { subgroup }\end{array}$ & Interview & $\begin{array}{l}\text { Baseline, week } \\
8 \text { and } 48^{\text {b }}\end{array}$ & $\begin{array}{l}\text { Investigator and } \\
\text { computerized touch } \\
\text { screen platform }^{c}\end{array}$ \\
\hline \multicolumn{6}{|c|}{$\begin{array}{l}\text { Abbreviations: MINI Mini-International Neuropsychiatric Interview, LES Life Events Scale, CTQ Childhood Trauma Questionnaire, SSQ Social Support Questionnaire, } \\
\text { SCSQ Simplified Coping Style Questionnaire, EPQ Eysenck Personality Questionnaire, HAMA Hamilton Anxiety Scale, HRSD } 17 \text { 17-Item Hamilton Rating Scale for Depres- } \\
\text { sion, CGIS Clinical Global impression Scale, CPT-IP Continuous Performance Test-Identical Pairs, AVFSS Animal Verbal Fluency Scale, CTTland II=Color Trial Test I and II, BACS } \\
\text { the Brief Assessment of Cognition in Schizophrenia, BVNT-R Brief Visual Memory Test-Revised, HVLT-R Hopkins Verbal Learning Test-Revised, SCWT Stroop Color Word Test } \\
\text { a Except "MDD course" for } 1200 \text { MDD patients only, other data of this domain would be collected for all participants } \\
\text { b For 300-Healthy controls subgroup, cognitive function data would only be collected at baseline } \\
\text { "Except for the data of CPT-IP collected using a standardized computerized touch screen platform, other data of this domain would be collected by the } \\
\text { trained investigator }\end{array}$} \\
\hline
\end{tabular}

\section{Sample size, power and effect size}

The first goal of this study is to verify the role of heritability and environment in the onset of MDD and identify a number of characteristics for defining the subtypes of MDD. The study design for this part is a case-control study. The sample size has been selected to detect small effects for factors at odds ratio 1.5 , if the proportion of factors in control group more than $10 \%$, with a power of 0.85 and a two-tailed significance level at 0.05 and the number of case equals control's, a total of 1042 MDD patients and 1042 healthy controls are intended to enroll. In view of $15 \%$ data missing, a total of 1200 MDD patients and 1200 healthy controls should be included.

The second goal of this study is to identify potential indicators to assist MDD diagnosis. The study design for this part is a diagnosis test study. The sample size has been selected to detect small effects for indicators at area under receiver operating characteristic curve more than 0.6 , with a power of 0.90 and a two-tailed significance level at 0.05 and the number of case is twice of control's, a total of 260 MDD patients and 130 healthy controls are intended to enroll. In view of $15 \%$ data missing, a total of 300 MDD patients and 150 healthy controls should be included. To confirm the performance of those indicators, another 300 MDD patients and 150 healthy controls should be included.

The third goal of this study is to identify factors which are associated with treatment outcomes of SSRIs on MDD. The study design for this part is a longitudinalbased case-control study. The sample size has been selected to detect small effects for factors at odds ratio 1.7, if the proportion of factors in control group more than $30 \%$, with a power of 0.82 and a two-tailed significance level at 0.05 and the number of case equal control's (half of MDD patients who receive one of 6 SSRIs in this study would have treatment response at the end of week 8). A total of 520 MDD patients are intended to enroll. In view of $13 \%$ data missing, a total of 600 MDD patients should be included.

\section{Data management}

The data management center, basing on Java ezweb using $\mathrm{B} / \mathrm{S}$ structure, has developed a web-based data system for data entering, checking and storage. After the paper-based questionnaire completed and checked, the site typist, with the typist's ID and password, will input the data into the web-based data system. The system, using the regular express method to control the input 
format for different data type, will do the basic format and logic check when the typist inputting the data and will give tips to the typist if the data out of the logic range. After passing the system check, the typist will clicked "submit" and then all the entered original data will be transferred to database server and labeled questionable. The CRA, with the checker's ID and password, will then review the questionable data, mark and write down their questions about the original data on the system and inform the researcher and the site typist to answer or modify the data, and then submit the reviewed data. After the reviewed data submitted, the data management center will lock and store the data in the final database using SQL Server 2008. The data system and database are installed in a host server which is collocated in an ISP server center and a special engineer is responsible for daily maintenance. Only having the approval of the executive management team, the engineer would export the database for the applicant. All data is deidentified using a unique ID number. The source documents are retained by each site and will be archived 10 years beyond study completion.

\section{Data preprocessing and statistical analysis}

Systematically data cleaning and quantification will be done before analysis. In the process of data preprocessing, the most import step is to handle missing data. In this study, missing data will be filled with average value of same label samples. In view of distance calculation, techniques of data normalization and standardization will be used.

The statistical analyses would be performed using Stata 10.0 soft-ware. The details of analytic approach for each study aim are as follows:

1. Verify the role of heritability and environment in the onset of MDD and define the subtypes of MDD according to the influence of heritability and environment on its development.

According to classification hypothesis, significance test for the classified characteristics of 1200-MDD patients and1200-Healthy controls would be done. The confounding factors would be adjusted using regression models. The interaction between heritability and environment on the onset of MDD would be evaluated using logistic regression. Cluster analysis, K Nearest Neighbor and K-means algorithms would be used to analyze the demographic information, family history of psychiatric diseases, life events in the past year, adverse childhood experience, social support, coping style and personality trait and to explore if MDD would be classify several subtypes according to the influence of heritability and environment on its development. For the sake of further distinguish between this high dimensional data, fuzzy clustering and spectral clustering would be employed in this trail to distinguish subtypes of MDD.
2. Identify potential indicators to assist MDD diagnosis basing on the baseline information, neuropsychological assessment and biological markers.

The characteristics difference of 600-MDD patients subgroup and 300 -Healthy controls subgroup will be analyzed. The correlation coefficient of each characteristic would be calculated and redundant features would be removed according to the experience of the clinical experts. Using logistic regression model to adjust the confounding factors, the effect of the rest factor set on outcome would be assessed and the main feature would be selected according to the weight coefficient. The principal component analysis and decision tree would be used to realize dimensionality reduction if the factor set is still large. To discriminate MDD patients from healthy controls, various classifiers, including support vector machines, decision tree, logistic regression and boosting, will be employed to cater to the demand of current situation. Significance test would be done for the selected factors and a characteristic will be considered an indicator if the p-value is $<0.05$. Half of $600-$ MDD patients subgroup and 300-Healthy controls subgroup would be used to train the model, and the rest half would be used to validate the model. The final indicators would be determined according to the best one model.

3. Identify overall predictors of treatment effectiveness on MDD after up to 8 weeks of SSRIs treatment and explore the predictors on MDD prognosis in the following two years.

Comparing with the baseline, according to the rate of decrease in HRSD17 at the end of 8-week, 600-MDD participants would be divided into effective group (the rate of decrease in HRSD17 $\geq 50 \%$ ) and ineffective group (the rate of decrease in HRSD17<50\%). The characteristics difference of the two groups will be analyzed. The correlation coefficient of each characteristic would be calculated and redundant features would be removed according to the experience of the clinical experts. Using logistic regression model to adjust the confounding factors, the effect of the rest factor set on outcome would be assessed and the main feature would be selected according to the weight coefficient. The principal component analysis would be used to realize dimensionality reduction if the factor set is still large. To predict treatment effectiveness, various classifiers, including typical classifiers such as support vector machines, decision tree, logistic regression and boosting and more powerful neural network, will be employed to cater to the demand of current situation. Significance test would be done for the selected factors and a characteristic will be considered a predictor if the $\mathrm{p}$-value is $<0.05$. The final predictors would be determined according to the best one model. The analysis plan for exploring the predictors on MDD prognosis in the following two years is similar to aforementioned. 


\section{Ethical issues}

This study had been approved by the Ethic Committee of Peking University Sixth Hospital (approval NO. 201329-1). Written informed consent was obtained from each subject prior to undertaking any study-related procedures. They will be informed that they can withdraw from the study at any time without any negative consequence. To conform to data protection and freedom of information acts, all data will be stored securely and anonymised wherever possible. No published material will contain identifiable patient information. The study procedure is being monitored and is being re-assessed annually by the Ethic Committee of Peking University Sixth Hospital.

\section{Discussion}

This study, a longitudinal, multi-stage and multi-center study, aims to verify the role of heritability and environment in the onset of MDD, define the subtypes of MDD, identify indicators to assist MDD diagnosis and assess predictors of treatment effectiveness of SSRIs on MDD. MDD may be divided into 3 types: heritability origin, stress origin and others. Potential indicators for diagnosis and predictors for treatment effectiveness may include demographic characteristics, stress events, clinical features, cognitive function, genotypes and peripheral biomarkers. Participants are recruited from clinical and academic sites to assemble a broadly inclusive and representative population. Thus, the study results should be widely generalization.

For different cause of MDD, the treatment response of antidepressants on MDD is usually various. To improve the treatment effectiveness, it is probably meaningful to divide MDD into different subtypes basing on its origin. To date, studies have typically examined one candidate marker at a time for assisting MDD diagnosis or predicting antidepressant response. By using standardized assessments to assess multiple candidate markers in the same study and same patients, this study provides enhanced statistical power to identify indicators to assist MDD diagnosis and assess predictors for antidepressant response. Promising preliminary empirical results coupled with recent developments in statistical methodology suggest that paradigms could be developed to provide useful clinical decision support in personalized treatment selection [47]. There will also be other strengths to this study, including strict quality control procedure, validated assessments by trained investigators, appropriate statistical analysis plan.

This study will have some limitations. First, brain imaging data was not collected owing to financial limit. Neuroimaging studies have demonstrated that brain imaging techniques could also assist to predict treatment outcomes of MDD [30, 48, 49]. On the other side, brain imaging techniques are quite complex and expensive at present and so it may be not suitable as a predictor for treatment response, especially in developing countries and regions. In this light, the predictors for treatment response on MDD studied in this study would be easily generalized. Second, the treatment for MDD patients is decided and adjusted by their attending physicians and they will be selected into this study if they meet the inclusion criteria. To some extent, the MDD subjects probably mirror clinical practice in a representative spectrum of MDD patients.

\section{Conclusion}

MDD patients usually display a wide variation in clinical symptoms and sign, while the diagnosis of MDD is relatively subjective. A about half of MDD patients fail to respond adequately to the first antidepressant. The development of neurobiology and statistical methodology make it sense to combine a set of possible factors for assisting the diagnosis and improving treatment effectiveness of MDD. The results of this study should provide strong and suitable evidence for enhancing the accuracy of MDD diagnosis and promoting personalized treatment for MDD in clinical practice.

\section{Trial status}

The recruitment of participants started in December 2013 and will stop at December 2016.

\section{Role of the funding source}

This project is funded by the National Key Basic Research Program of China (No.2013CB531305). The study protocol has undergone peer-review by the funding body.

\section{Acknowledgements}

The authors thank all researchers and investigators for their participation in this multicenter study: Pro Jing Wei, Pro Gang Zhu, Pro Kerang Zhang, Pro Xueyi Wang, Pro Hongjun Tian, Pro Qiaoling Chen, Dr Nan Zhang and their teams. The authors thank Beijing Venture-Sunshine Pharmaceutical T.Co.,Ltd, Dr Xuemei Song and her team for assisting the execution of this program. The authors thank Pro Jianguo Chen for guiding the program.

\section{Funding}

National Key Basic Research Program of China (No.2013CB531305).

Availability of data and materials

Not applicable.

\section{Authors' contributions}

LXZ drafted this paper which was added to and modified by all other authors. YX, STM,WG,WHL, LQ, WJ, SYA, HCQ and LXZ participated in the design of the study. LXZ and WJ participated in the coordination between all the centers and ethics formalities. $\mathrm{HY}$ and $\mathrm{JH}$ contributed to data management and the analytic strategy. All authors read and approved the final manuscript.

\section{Competing interests}

The authors declare that they have no competing interests.

Consent to publish

Not applicable. 


\section{Ethics approval and consent to participate}

This study had been approved by the Ethic Committee of Peking University Sixth Hospital (approval NO. 2013-29-1). Written informed consent was obtained from each subject prior to undertaking any study-related procedures.

\section{Author details Beijing, China. \\ Received: 1 June 2016 Accepted: 1 July 2016 \\ Published online: 15 July 2016}

'Peking University Sixth Hospital (Institute of Mental Health), Beijing, China. ${ }^{2}$ National Clinical Research Center for Mental Disorders \& Key Laboratory for Mental Health, Ministry of Health, Peking University, Beijing, China. ${ }^{3}$ Beijing Anding Hospital, Capital Medical University, Beijing, China. ${ }^{4}$ National Engineering Research Center for Software Engineering, Peking University,

\section{References}

1. World Health Organization. Depression. http://www.who.int/mediacentre/ factsheets/fs369/en/. Accessed on April 22, 2015

2. Ustun TB, Ayuso-Mateos JL, Chatterji S, Mathers C, Murray CJ. Global burden of depressive disorders in the year 2000. Br J Psychiatry. 2004;184:386-92.

3. Ferrari AJ, Charlson FJ, Norman RE, Patten SB, Freedman G, Murray CJ, et al. Burden of depressive disorders by country, sex, age, and year: findings from the global burden of disease study 2010. PLoS Med. 2013;10, e1001547.

4. Greenberg PE, Kessler RC, Birnbaum HG, Leong SA, Lowe SW, Berglund PA, et al. The economic burden of depression in the United States: how did it change between 1990 and 2000. J Clin Psychiatry. 2003;64:1465-75.

5. Phillips MR, Zhang J, Shi Q, Song Z, Ding Z, Pang S, et al. Prevalence, treatment, and associated disability of mental disorders in four provinces in China during 2001-05: an epidemiological survey. Lancet. 2009;373:2041-53.

6. Sullivan PF, Neale MC, Kendler KS. Genetic epidemiology of major depression: review and meta-analysis. Am J Psychiatry. 2000;157:1552-62.

7. Keers R, Uher R. Gene-environment interaction in major depression and antidepressant treatment response. Curr Psychiatry Rep. 2012;14:129-37.

8. Kendler KS, Kessler RC, Walters EE, MacLean C, Neale MC, Heath AC, et al. Stressful life events, genetic liability, and onset of an episode of major depression in women. Am J Psychiatry. 1995:152:833-42.

9. Van Loo HM, De Jonge P, Romeijn JW, Kessler RC, Schoevers RA Data-driven subtypes of major depressive disorder: a systematic review. BMC Med. 2012;10:156

10. Fava M, Kendler KS. Major Depressive Disorder. Neuron. 2000;28:336-41.

11. Lin CH, Huang CJ, Liu SK. Melancholic features in inpatients with major depressive disorder associate with differential clinical characteristics and treatment outcomes. Psychiatry Res. 2016;238:368-73.

12. Papakostas $\mathrm{Gl}$, Larsen $\mathrm{K}$. Testing anxious depression as a predictor and moderator of symptom improvement in major depressive disorder during treatment with escitalopram. Eur Arch Psychiatry Clin Neurosci. 2011:261:147-56

13. Iosifescu DV, Clementi-Craven N, Fraguas R, Papakostas Gl, Petersen T, Alpert JE, et al. Cardiovascular risk factors may moderate pharmacological treatment effects in major depressive disorder. Psychosom Med. 2005;67:703-6.

14. Lopresti AL, Maker GL, Hood SD, Drummond PD. A review of peripheral biomarkers in major depression: the potential of inflammatory and oxidative stress biomarkers. Prog Neuropsychopharmacol Biol Psychiatry. 2014;48:102-11.

15. Schmidt HD, Shelton RC, Duman RS. Functional biomarkers of depression: diagnosis, treatment, and pathophysiology. Neuropsychopharmacology. 2011;36:2375-94

16. Valkanova V, Ebmeier KP, Allan CL. CRP, IL-6 and depression: a systematic review and meta-analysis of longitudinal studies. J Affect Disord. 2013;150:736-44

17. Blume J, Douglas SD, Evans DL. Immune suppression and immune activation in depression. Brain Behav Immun. 2011:25:221-9.

18. Dowlati Y, Herrmann N, Swardfager W, Liu H, Sham L, Reim EK, et al. A meta-analysis of cytokines in major depression. Biol Psychiatry. 2010;67:446-57.

19. Papakostas Gl, Shelton RC, Kinrys G, Henry ME, Bakow BR, Lipkin SH, et al. Assessment of a multi-assay, serum-based biological diagnostic test for major depressive disorder: a pilot and replication study. Mol Psychiatry. 2013:18:332-9.
20. Yamamori H, Ishima T, Yasuda Y, Fujimoto M, Kudo N, Ohi K, et al. Assessment of a multi-assay biological diagnostic test for mood disorders in a Japanese population. Neurosci Lett. 2016;612:167-71.

21. Trivedi MH, Rush AJ, Wisniewski SR, Nierenberg AA, Warden D, Ritz L, et al. Evaluation of outcomes with citalopram for depression using measurement-based care in STAR*D: implications for clinical practice. Am J Psychiatry. 2006;163:28-40.

22. Hennings JM, Owashi T, Binder EB, Horstmann S, Menke A, Kloiber S, et al. Clinical characteristics and treatment outcome in a representative sample of depressed inpatients - findings from the Munich Antidepressant Response Signature (MARS) project. J Psychiatr Res. 2009;43:215-29.

23. Perlis $\mathrm{RH}$. A clinical risk stratification tool for predicting treatment resistance in major depressive disorder. Biol Psychiatry. 2013;74:7-14.

24. Rush AJ, Wisniewski SR, Warden D, Luther JF, Davis LL, Fava M, et al. Selecting among second-step antidepressant medication monotherapies: predictive value of clinical, demographic, or first-step treatment features. Arch Gen Psychiatry. 2008:65:870-80.

25. Kloiber S, Ising M, Reppermund S, Horstmann S, Dose T, Majer M, et al. Overweight and obesity affect treatment response in major depression. Biol Psychiatry. 2007;62:321-6.

26. Papakostas Gl, Fava M. Predictors, moderators, and mediators (correlates) of treatment outcome in major depressive disorder. Dialogues Clin Neurosci. 2008;10:439-51.

27. Licinio J, Dong C, Wong ML. Novel sequence variations in the brain-derived neurotrophic factor gene and association with major depression and antidepressant treatment response. Arch Gen Psychiatry. 2009;66:488-97.

28. Leuchter AF, Cook IA, Hamilton SP, Narr KL, Toga A, Hunter AM, et al. Biomarkers to predict antidepressant response. Curr Psychiatry Rep. 2010;12:553-62.

29. Frieling $\mathrm{H}$, Tadic A. Value of genetic and epigenetic testing as biomarkers of response to antidepressant treatment. Int Rev Psychiatry. 2013;25:572-8.

30. Lener MS, losifescu DV. In pursuit of neuroimaging biomarkers to guide treatment selection in major depressive disorder: a review of the literature. Ann N Y Acad Sci. 2015;1344:50-65

31. Polyakova M, Stuke K, Schuemberg K, Mueller K, Schoenknecht $P$, Schroeter ML. BDNF as a biomarker for successful treatment of mood disorders: a systematic \& quantitative meta-analysis. J Affect Disord. 2015;174:432-40.

32. Huang TL, Lin CC. Advances in biomarkers of major depressive disorder Adv Clin Chem. 2015;68:177-204.

33. Thase ME. Using biomarkers to predict treatment response in major depressive disorder: evidence from past and present studies. Dialogues Clin Neurosci. 2014;16:539-44.

34. Lim SW, Won HH, Kim H, Myung W, Kim S, Kim KK, et al. Genetic prediction of antidepressant drug response and nonresponse in Korean patients. PLoS ONE. 2014:9, e107098.

35. Lin JY, Jiang MY, Kan ZM, Chu Y. Influence of 5-HTR2A genetic polymorphisms on the efficacy of antidepressants in the treatment of major depressive disorder: a meta-analysis. J Affect Disord. 2014;168:430-8.

36. Zou YF, Ye DQ, Feng XL, Su H, Pan FM, Liao FF. Meta-analysis of BDNF Val66Met polymorphism association with treatment response in patients with major depressive disorder. Eur Neuropsychopharmacol. 2010;20:535-44

37. Niitsu T, Fabbri C, Bentini F, Serretti A. Pharmacogenetics in major depression: a comprehensive meta-analysis. Prog Neuropsychopharmacol Biol Psychiatry. 2013;45:183-94.

38. Sung SC, Wisniewski SR, Balasubramani GK, Zisook S, Kurian B, Warden D, et al. Does early-onset chronic or recurrent major depression impact outcomes with antidepressant medications? A CO-MED trial report. Psychol Med. 2013;43:945-60.

39. Zhang ZJ. Manual of Behavioral Medicine Scales. Beijing: Chinese Medicine Electronic Audio and Video Press; 2005

40. Si TM, Shu L, Dang WM, Su YA, Chen JX, Dong WT, et al. Evaluation of the reliablility and validity of Chinese version of the Mini-International Neuropsychiatric Interview in patients with mental disorders. Chin Ment Health J. 2009;23:493-503.

41. American Psychiatric Association. Diagnostic and statistical manual of mental disorders. 4th ed. Washington: American Psychiatric Publishing; 2000.

42. Wang XD, Wang XL, Ma H. Manuals of Mental Health Assessment Scales: the Expanded Edition. Beijing: Chinese Mental Health Journal Publishing; 1999. 
43. Yu X. Handbook of MATRICS Consensus Cognitive Battery Chinese Norm. Beijing: Peking University Medical Press; 2014.

44. International HapMap Project. http://hapmap.ncbi.nlm.nih.gov/. Accessed 11 May 2016

45. dbSNP. http://www.ncbi.nlm.nih.gov/snp/. Accessed 11 May 2016.

46. Lee PH, Shatkay H. F-SNP: computationally predicted functional SNPs for disease association studies. Nucleic Acids Res. 2008;36:D820-4.

47. Kessler RC, Van Loo HM, Wardenaar KJ, Bossarte RM, Brenner LA, Ebert DD, et al. Using patient self-reports to study heterogeneity of treatment effects in major depressive disorder. Epidemiol Psychiatr Sci. 2016;1-15.

48. Sikora M, Heffernan J, Avery ET, Mickey BJ, Zubieta JK, Pecina M. Salience Network Functional Connectivity Predicts Placebo Effects in Major Depression. Biol Psychiatry Cogn Neurosci Neuroimaging. 2016;1:68-76.

49. Vasavada MM, Leaver AM, Espinoza RT, Joshi SH, Njau SN, Woods RP, et al. Structural connectivity and response to ketamine therapy in major depression: A preliminary study. J Affect Disord. 2016;190:836-41.

Submit your next manuscript to BioMed Central and we will help you at every step:

- We accept pre-submission inquiries

- Our selector tool helps you to find the most relevant journal

- We provide round the clock customer support

- Convenient online submission

- Thorough peer review

- Inclusion in PubMed and all major indexing services

- Maximum visibility for your research

Submit your manuscript at www.biomedcentral.com/submit
Biomed Central 